zugeschrieben. Diese Erklärung gilt aber nicht für die Emphyseme, die ohne nennenswerte Bronchitis zustande kommen. Wir kommen deshalb um die Erklärung der Zirkulationsstörung durch den Untergang von Capillaren doch nicht herum.

Man hat in letzter Zeit versucht, die Zirkulationsverhältnisse beim Lungenemphysem mit Hilfe neverer gasanalytischer Methoden zu untersuchen. Bei den. Gesunden gelingt es, den Sauerstoff- und Kohlensäuregehalt im Blute der Lungenarterien und -Venen zu berechnen, indem man die Zusammensetzung der Alveolarluft in verschiedenen Respirationsphasen bestimmt. Beim Emphysem versagen aber diese Methoden vollständig, weil es kaum gelingt, eine gleichmäßige Durchmischung der Lungenluft zu erreichen, wie wir schon erwähnt haben. Einzig die direkte Analyse des Blutes aus einer Arterie gibt uns Aufschluß über die Zusammensetzung des Blutes in den Lungenvenen, also über die Arterialisation in den Lungen. Der erste, der Blut aus der Arteria radialis am lebenden Menschen analysiert hat, war HÜRTER, und dieser hat auch schon beim Emphysem Untersuchungen vorgenommen. In den letzten Jahren ist diese Methode von Amerikanern aufgenommen und angewandt worden. Es hat sich dabei ergeben, was eigentlich zu erwarten war, daß die Arterialisation des Blutes in der Emphysemlunge in erster Linie von der Bronchitis abhängt, die je nach ihrer Ausdehnung und Lokalisation den Gasaustausch hindern kann. Dazu kommt aber noch die gleich $\mathrm{zu}$ besprechende ungenügende Ventilation vieler Alveolen.

Ober die Dyspnöe beim. Emphysem liegt eine Reihe von Arbeiten vor. Es ist schon lange bekannt, daß die Vitalkapazität bei dieser Krankheit vermindert ist, was mit der Erweiterung der Lunge, aber auch mit der Thoraxstarre erklärt werden kann. Diese Verminderung der Vitalkapazität muß bei tiefen Atemzügen hinderlich sein. Dazu kommt aber beim Emphysematiker noch etwas anderes. Wie ich zusammen mit SCHÜTZE nachweisen konnte, atmet der Emphysematiker tiefer als ein Gesunder. REINHARDT hat das bestätigt und gefunden, daB der $\mathrm{CO}_{2}$-Prozentgehalt der Exspirationsluft beim Emphysematiker herabgesetzt ist. Das kommt offenbar daher, daß die eingeatmete Luft sich beim Emphysematiker in der Lunge schlecht verteilt. Dadurch entsteht Kohlensäureanhäufung in den schlecht ventilierten Alveolen. Durch eine verstärkte Atmung können die gut zugänglichen Alveolen so stark gelüftet werden, daß der Kohlensäuregehalt im Blut ihrer Capillaren stärker herabgesetzt wird als normal und $\mathrm{da} B$ das Mischblut in den Lungenvenen einen normalen Kohlensäuregehalt bekommt. Anders liegt es mit dem Sauerstoff. Das Hämoglobin wird mit diesem schon bei der normalen Atmung nahezu gesättigt, und die stärkste Ventilation kann hier nichts mehr verbessern, sie bringt aber auch in den schlecht ventilierten Alveolen keinen normalen Sauerstoffgehalt mehr zustande, deshalb muß das Mischblut, also das Blut in den Lungenvenen und Körperarterien, zwar einen normalen Kohlensäuregehalt, aber einen herabgesetzten Sauerstoffgehalt aufweisen. Das hat MEAkins tatsächlich bei schweren Emphysemen gefunden, und er hat auch gezeigt, daß Sauerstoffatmung die Arterialisation des Blutes bessern kann. Wir haben also meherere Ursachen für die Arbeitsdyspnöe der Emphysematiker, nämlich die mechanische Hinderung der Atemexkursionen, die Vermehrung der Lungenventilation im Vergleich zum Gesunden bei gleichem Gaswechselbedarf und vielleicht auch den Sauerstoffmangel des Blutes, Dazu kommt in manchen Fällen noch die Zirkulationsstörung. Am wenigsten Bedeutung hat wohl der Sauerstoffmangel, aber er ist insofern wichtig, als er in manchen Fällen die Cyanose erklärt, die man gewöhnlich auf die Zirkulationsstörung bezicht. Nach neueren Untersuchungen muß man nämlich annehmen, daß Sauerstoffarmut des Blutes tatsächlich Cyanose erzengt. Allerdings gilt das in erster Linie für Fälle mit schwerer Bronchitis, und aus den Versuchen Meakins geht die Bedeutung der Bronchitis für das Verhalten der Blutgase deutlich hervor.

Auch für die Vitalkapazität hat die Bronchitis eine große Bedeutung. Man sollte denken, daß die Verminderung der Vitalkapazität in erster Linie dadurch bedingt ist, daß die Lungen sich nicht genügend entleeren können. Man müßte deshalb vorwiegend eine Verminderung der Reserveluft erwarten. Aus den Untersuchungen von Bruss und von BitTorf und ForschBACH geht aber hervor, daB immer noch eine, wenn auch geringe Reserveluft vorhanden ist und daB der Thorax bisweilen in seiner Einatmungsfähigkeit gleich beschränkt ist wie in seiner Ausatmungsfähigkeit, da $B$ also die Komplementärluft und die Reserveluft in ähnlicher Weise herabgesetzt sein können. Auch in den Versuchen der genannten Forscher zeigt sich der Einfluß der Bronchitis. Auch eine Untersuchung dieser Verhältnisse bei einzelnen Formen von Emphysemen dürftefür die Frage der Pathogenese von Interesse. sein.

Zum Schluß möchte ich nur ganz kurz darauf eingehen, ob die Therapie von den neueren Forschungen Nutzen ziehen kann. In erster Linie denken wir natürlich an die FREUnD sche Operation. Es ist unbestreitbar, daß sie die Exkursionsfähigkeit des Thorax bessern kann, selbst wenn die Rippenknorpeldegeneration nicht das Primäre ist. In der Tat sieht man auch in dieser Hinsicht deutliche Erfolge, wie die Zusammenstellungen von VON DEN VELDEN und von ROUBACHOW zeigen. Es ist aber auffallend, daß in der letzten Zeit nicht mehr sehr viel über die Operation verlautet ist. Ich kann selbst keinen Beitrag zu dieser Frage bringen, da es mir bisher erst gelungen ist, einen einzigen Patienten zur Operation zu überreden. Bei diesem war allerdings der Erfolg recht befriedigend. Die meisten Kranken wollen sich aber nicht operieren lassen, wenn sie keine Bronchitis haben, weil die Thoraxstarre allein keine genügenden Beschwerden macht und sie sich zu einer prophylaktischen Operation nicht entschließen können. Bei erheblicher Bronchitis habe ich den Vorschlag zu einer Operation noch nie gewagt, und wenn die Bronchitis sich besserte, wollten die Patienten nichts mehr davon wissen.

Massage, Gymnastik, Pneumatotherapie, Roßbachscher Atmungsstuhl und ähnliche Methoden, die die Exspiration befördern, scheinen mir hauptsächlich durch die Erleichterung der Expektoration und die Besserung der bronchitischen Beschwerden zu wirken. Es ist auch nicht anzunehmen, daB ein starrer Thorax oder eine erweiterte Lunge dauernd $z u-$ sammengepreßt werden könnte oder daß diese Zusammenpressung einen wesentlichen Erfolg bedeuten würde. Das Wichtigste für die Besserung der Beschwerden und für die Prophylaxe des Emphysems, sowie zur Verhinderung seiner Weiterentwicklung bleibt immer noch die Behandlung der Bronchitis, und wir müssen froh sein, wenn die Forschung den pathologischen Anatomen und Klinikern den Glauben an eine bronchitische Genese des Emphysems und an die Möglichkeit einer therapeutischen Einwirkung nicht nehmen kann.

\title{
ORIGINALIEN.
}

\section{DIE „AKTIVIERUNG“ DER KÖRPERZELLEN UND DER INFEKTIONSERREGER.}

Studien über Immunität und Virulenz.

Professor Dr. Wolfgaivg Weichardt, Erlangen.

Nachdem die Proteinkörpertherapie in den letzten Jahren besonders aktuell geworden ist, haben sich recht zahlreiche
Autoren auch mit ihrer theoretischen Begrïndung beschäftigt.

Zusammenfassungen über den neuesten Stand dieser Frage finden sich in der in Nordamerika erschienenen Monographie von W.F. Petersen ${ }^{1}$ ), dem Referat von A. Schittenimelm ${ }^{2}$ ) auf der 9. Versammlung der freien Vereinigung für Mikrobiologie I922 und in den Ergebnissen der Hygiene usw. Band V3).

Zunächst sei auf einige, vor allem den Praktiker interessierende prinzipielle Gesichtspunkte Bezug genommen: 
Daß man eigentlich schon seit sehr langer Zeit Proteinkörpertherapie trieb, ist in den letzten Jahren mehrfach betont worden. Schon bei den alten Ärzten, deren auf scharfe Beobachtungsgabe begründete Empirie bekannt ist, sehen wir oft eine überraschend gute Einstellung auf die Bedürfnisse der neuzeitlichen Proteinkörpertherapie, besonders bei den Transfusoren, welche das Blut eines Tieres auf den Menschen übertrugen.

Immerhin kann man erst von einer bewußt betriebenen Proteinkörpertherapie sprechen, nachdem mit den Mitteln neuzeitlicher naturwissenschaftlicher Erkenntnis die Gründe der Wirkung oder des Versagens der Proteinkörpertherapie durchforscht werden [A. Schittenhelm ${ }^{2}$ )].

Es kann auch von einer bewußt betriebenen Proteinkörpertherapie keine Rede sein, wenn neuere Autoren, wie das schon die alten Trazsfusoren getan hatten, die Wirkung von einzelnen Erscheinungen aus beurteilten, und sie davon abhängig zu machen suchen, so von dem Fieber, der Leukocytose, der Entzündung, der Antikörperbildung u. a. m.

Wie die Beurteilung von einer Erscheinung*) aus niemals zur Grundlage einheitlicher Erfassung eines derartig innerlich zusammenhängenden, äußerlich so verschiedenartig erscheinenden Gebietes dienen kann, so kann eine solche Grundlage auch nicht darin gefunden werden, daß eine einzige Ursache für die bei Proteinkörpertherapie beobachteten Symptome verantwortlich gemacht und zur Namengebung herangezogen wird (kolloidoklastische Therapie, Kolloidtherapie, Osmosetherapie, Reiztherapie u. v. a.m.). diesem Gebiete nicht entspricht*). Was wir bei der Proteinkörpertherapie sehen, ist lediglich eine Reaktionsänderung des Organismus, und was wir in den meisten Fällen erreichen wollen, ist eine Leistungssteigerung nach einer bestimmten oder nach den verschiedensten Richtungen hin. Auch kann natürlich eine Erregung hemmender Nerven zu einer Leistungsminderung eines Organes führen.

Besonders interessant sind die Aktivierungen, welche sich darin äußern, daß die Reizschwelle für bekannte spezifisch wirkende Gifte verändert wird. In der letzten Zeit hat FREUND $^{5}$ ) über Steigerungen der Adrenalinwirkungen auf den Blutdruck und der Pilocarpinwirkung auf die Speichelsekretion berichtet. Untersuchungen von STARKENSTEIN ${ }^{6}$ ) sind hier zu nennen: dieser fand nach Milchinjektion erhöhte Widerstandsfähigkeit gegen Strychnin. Nach Einspritzung von Albumosen und Peptonen war die Phenolwirkung abgeschwächt. Verhältnismäßig zahlreich sind klinische Angaben über Beförderungen von Arzneimittelwirkungen durch Proteinkörperinjektionen. So teilt z. B. im Jahre I9I7 EDELMANN $^{7}$ ) günstige Beeinflussung der Salicylmedikationen durch Proteinkörper mit. KYRLF ${ }^{8}$ ) fand bei Behandlung der Syphilis durch Injektionen von Milch und Quecksilber, daß geringere Mengen von Quecksilber als früher benötigt wurden, um die primären und sekundären Erscheinungen zum Verschwinden zu bringen, sowie den positiven Ausfall der Wassermannschen Reaktion in einen negativen zu verwandeln.

Es sei hier auf ein früher bereits von mir aufgestelltes Schema verschieden möglicher Arten von Leistungssteigerun-

Leistungssteigerung ist möglich:

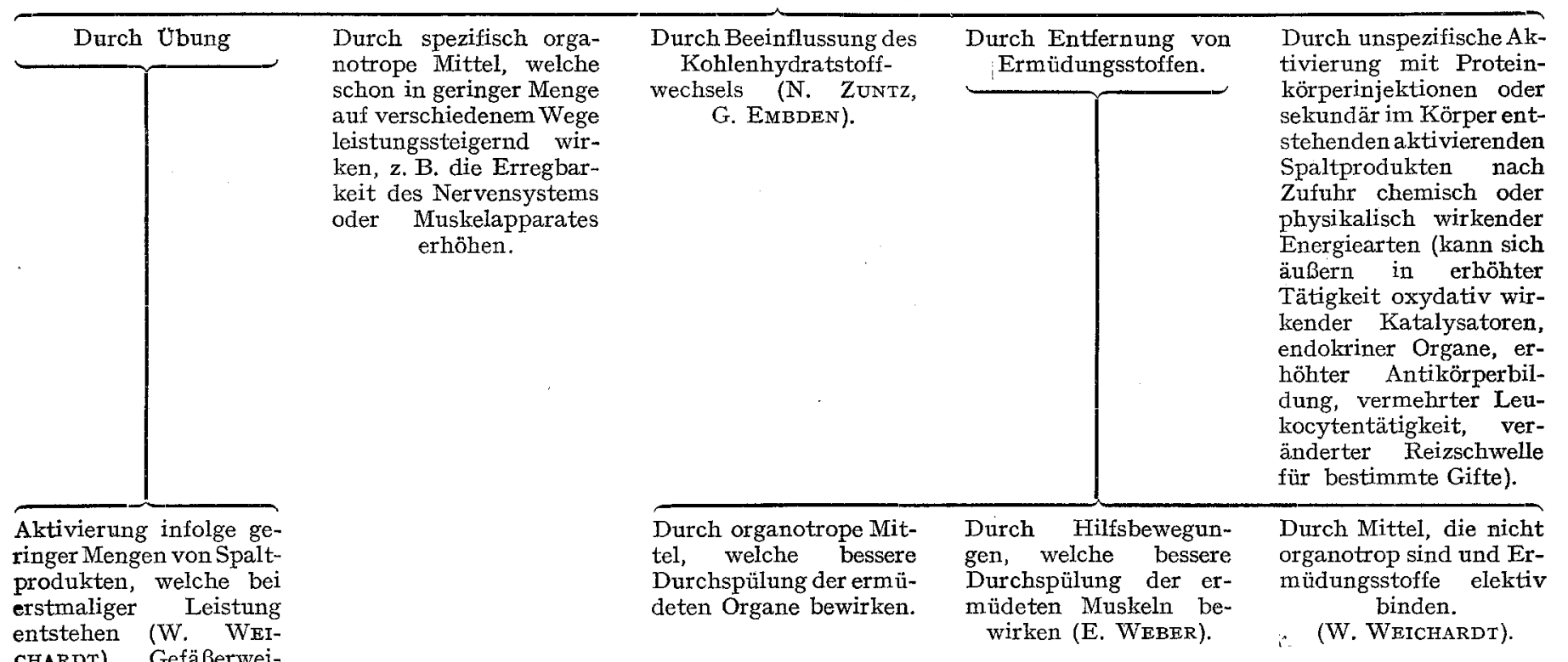

CHARDT). Gefäßerwei-

terung (HENDERSON-

LOEWI, E. WEBER).

Vermehrung der Form-

$$
\text { elemente. }
$$

Eine einheitliche Beurteilung wird nur erreicht, wenn wir ein allgemeines Prinzip, einen wirklich allgemeinen Begriff mit Erklärungswert in den Vordergrund der Betrachtung stellen, wodurch alle in Frage kommenden Symptome einheitlich zusammengefaßt werden. Als solcher hat sich, wie aus der Literatur leicht ersichtlich, der von mir eingeführte Begriff der Altivierung des Plasmas und der Zellen sehr bewährt. Hierunter darf nicht etwa, wie ich in Nr. 4 der Münch. med. Wochenschr. I922 des genaueren ausgeführt habe, eine einfache Reizung verstanden werden. Es wäre das eine durchaus einseitige Auffassung, die unseren Kenntnissen auf

*) In der letzten Zeit wexden allexdings die auf Heraushebung nur eines Symptomes gestellten Begriffe so weit gefaßt, daß die sonst einzuwendende Einseitigkeit gemildert wird und sich nicht viel mehr direkt dagegen sagen läßt. Ein so zu weit gefaßter Symptombegriff verliert aber bekanntlich an Prägnanz und damit für uns an Wert, so daß er dann als solcher ganz unbestimmt, gegenstandslos und überflüssig wird.

gen hingewiesen**), wobei von Fall zu Fall zu entscheiden ist, ob eine direkte Beeinflussung der Zellen des Erfolgsorganes vorliegt, oder ob die Leistungssteigerung indirekt zustande kommt durch humorale Vorgänge oder durch Beeinflussung der zentralen oder peripheren nervösen Apparate. $\mathrm{Zu}$ bemerken ist, daß selbstverständlich jede schematische Darstellung des Naturgeschehens nur ein lückenhaftes Bild der Wirklichkeit geben kann, da meist mehrere Ursachen ineinander greifen.

Die Vorgänge, welche zu dem gleichen Effekt der Leistungssteigerung führen, sind also im Gresamtorganismus des *) R. SCHMIDT, der als Kliniker zuerst auf diese Zusammenhänge hinwies und den Begriff der "Proteinkörpertherapie" aufstellte, faßt die Aktivierung richtig auf als ,Anderung der Gleichgew'ichtslage mit Neigung zur Hyperaltivität". Nach ihm wird durch diese Auffassung die Perspektive auf die Möglichkeit von Umstimmungen fehlerhaft eingestellter Gesamt- und Partialkonstitutionen eröffnet. Ergebn. d.

ges. Med. Bd. III ${ }^{4}$ ).
*; ) Ergebnisse d. Hyg., Balkteriol., Immunitäts-Forsch. u, exp. Therapie, 5, S. 32 r. 
Warmblüters außerordentlich vielgestaltig. Einfachere Lebewesen bieten demgegenüber oft leichter überblickbare Verhältnisse und vielfach bessere Möglichkeiten, den Gründen bestimmter Leistungssteigerungen nachzuspüren. Als verhältnismäßig gute Objekte für ein quantitatives Studium wählten wir Parasiten, die in bezug auf ihren Fermenthaushalt sehr dem Körper angepaßt sind. So erwiesen sich einzelne Streptokokkenstämme zum Studium leistungssteigernder Einflüsse auf die verschiedenen Fermentfunktionen als geeignet.

Bekanntlich vermögen diese auf Nährböden. wie sie $z$. B. von C. FRÄNKEL aus verhältnismäßig einfachen, chemisch definierbaren Bestandteilen zusammengesetzt worden sind, sich nicht wesentlich $\mathrm{zu}$ vermehren, auch wenn man diesen Nährböden noch reichlich einfachere N-haltige Gruppen, Kohlenhydrate und Salze zusetzt. Fügt man ihnen jedoch in geringen Mengen abiurete Extraktstoffe zu, welche in der unten beschriebenen Weise aus dem Tierkörper gewonnen werden, so findet sofort cin reichliches Wachstum statt und die feinere Analyse zeigt, daß sowohl die Kohlehydrate verdauenden Fermente als auch diejenigen, welche stickstoffhaltige Gruppen zerlegen und die, welche für das Wachstum in Frage kommen, in ihrer Tätigkeit angeregt werden. Auch die Giftbildung wird in bemerkenswerter Weise beeinflußt. Einige Beispiele von Versuchen, von denen wir in den letzten Jahren eine große Anzahl angestellt und veröffentlicht haben ${ }^{9}$, seien im folgenden beschrieben:

Die abiureten wirksamen Extraktstoffe aus dem Körper wurden folgendermaßen hergestellt:

Den entbluteten Tieren wurde Magen und Darm steril entfernt, in manchen Versuchen wurden sie mit Toluolwattebäuschen ausgestopft und in den Brutschrank verbracht. Sie wurden dann unter aseptischen Kautelen zerkleinert, mit der dreifachen Menge Alkohol 20 Minuten gekocht, das filtrierte Extrakt im FaustHeim Apparat getrocknet, mit Wasser ausgezogen, die wasserunlöslichen Substanzen durch Zentrifugieren und Filtrieren entferntund das Filtrat vor dem Eintrocknen genau neutralisiert (Lackmus). Bei sorgfältigem Arbeiten waren die so erhaltenen wasserlöslichen Substanzen abiuret. Bei weiterem Reinigen mit Phosphor-Wolframsäure auf dem üblichen Wege ging der größte Teil der wirksamen Stoffe in das Filtrat über. Weiter konnten die Versuche aus Mangel an Material nach dieser Richtung nicht getrieben werden. Verwendet wurden zunächst die abiureten alkoholwasseriöslichen Extrakte. Diese wurden in Normosal gelöst, das ein für unsere Parasiten ohne weiteres nicht verwertbares physiologisches Gemisch anorganischer Salze und abiureter organischer Verbindungen enthält (Glykokollcalcium u. a.). Um auf alleFälle genügend N- und Kohlenhydratquellen zu haben, wurden in vielen Fällen rproz. Natrium asparaginicum und Iproz. Glyzerin zugesetzt. Die Röhrchen wurden dann sterilisiert, und zwar erweist es sich als vorteilhaft, die schwächeren Extraktverdünnungen, bei deren Verarbeitung bereits möglichst steril vorgegangen werden muß, kurz in strömenden Dampf zu bringen. Bei den stärkeren Extraktkonzentrationen treten Fällungen auf. Es wurde in diesem Falle durch das KNORRsche Kieselgurweichfilter steril filtriert. Die Röhrchen wurden sodann in ganz gleicher Weise mit der gleichen Óse einer I8stündigen, gleichmäßig verteilten Streptokokkenbouillonkultur beimpft, sodann nach $16-17$ stündigem Bebrüten bei $37^{\circ}$ Blutagarzählplatten*) gegossen.

Aus Tabelle I geht hervor, daß schon geringe Mengen von Extrakt, einem flüssigen Nährboden zugesetzt, auf dem sonst Streptokokken in I5 Stunden sich nicht vermehren, eher vermindern, ein weit über die Versuchsfehlergrenze hinausgehendes Streptokokkenwachstum veranlassen. Aus der Tabelle ist ferner zu ersehen, daß die Extraktstoffe, welche die Vermehrung der Parasiten bedingen, durch zostündiges Verweilen der Organe bei $37^{\circ}$ deutlich vermehrt wurden. Setzt man den zerkleinerten Organen verdünnte Milchsäure zu und läßt dieselben I $_{5}$ Stunden im Eisschrank, so bekommt man, wie aus Tabelle II hervorgeht, ebenfalls bedeutend mehr wachstumsfördernde Substanzen, als wenn

*) Zu diesen Versuchen ist zu bemerken, daß sie ein AusfluB jahrelang fortgeführter Technik'sind, daß insbesondere die Gewinnung der Zählresultate ein besonderes Einarbeiten nötig macht. Nur Platten mit mittlerem. Keimgehalt sind als Zählplatten: zu verwenden. Nur aus großen Versuchsreihen sind Schlüsse zu zięen. Gelegentliche Versager kommen auch dem Geübten vor. Eine ausführliche Veröffentlichung aller Versuchsresultate erfolgt zusammenfassend an anderer Stelle, (WOLF. GANG WEICHARDT, IRMGARD v. SODEN, MAXIMILIAN KNORR, Erg. d. Hyg.).

man ohne Milchsäure nach I5stündigem Verweilen im Eisschrank die Organe auf Extrakt in der beschriebenen Weise verarbeitet*). Wie ich früher zeigte, ist das nach verschiedenen Richtungen von Interesse. Wissen wir doch, da $B$ bei hochgradiger Ermüdung und Störung im Kohlenhydratstoffwechsel besonders septicämische Prozesse zu fürchten sind.

Tabelle I.

Wachstumszahlen mit wässerigen Auszügen von Alkoholextrakten aus Meerschweinchenorganen.

Streptococcus, haemolyt.

\begin{tabular}{|c|c|}
\hline $\begin{array}{l}\text { Grad der Verdünnung des Ex- } \\
\text { traktes in } 5 \mathrm{ccm} \text { Normosal }\end{array}$ & Zahl der Kolonien \\
\hline 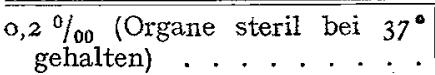 & 3194862 \\
\hline $0,2 \%$ (Organe frisch verarbertet) & I I 79788 \\
\hline Kontrolle ohne Extrakt, be brütet & I7 400 \\
\hline $\begin{array}{c}\text { Kontrolle ohne Extrakt, unbe- } \\
\text { brütet. . . . . . . }\end{array}$ & I I 400 \\
\hline
\end{tabular}

Wachstumszahlen von Streptokokken in wässerigen Auszügen von Alkoholextrakten aus Meerschweinchenorganen, die z. T. mit $\mathrm{n} / 10$ Milchsäure versetzt wurden.

Streptococcus haemolyt.

Grad der Verdünnung des Extraktes in $5 \mathrm{ccm}$ Normosal mit I proz. Glycerin

$0,2 \%$ (mit $\mathrm{n} / 10$ Milchsäure ver-

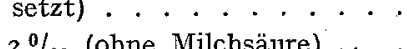
Kontrolle ohne Extrakt, bebrütet. . . . . . . . . Kontrolle ohne Extrakt, unbebrütet. . . . . . . .

Endlich infizierten wir Mäuse mit Friedländerbacillen, töteten die schwer erkrankten Tiere kurz vor dem Exitus und verarbeiteten sie sofort in der oben beschriebenen Weise. Gleichzeitig wurde eine gleiche Menge Mäusematerial ganz gesunder Tiere**) ebenso verarbeitet. Aus Tabelle III geht hervor, daß auch durch Infektion mit Friedländer bei Mäusen eine erhebliche Vermehrung der Streptokokken aktivierenden Spaltprodukte verursacht wird. Diese werden sowohl aus den Körpergeweben als aus den Septikämieerregern, die sich massenhaft im Körper verbreitet hatten, stammen.

Tabelle III.

Wachstumszahlen mit wässerigen Auszügen von Alkoholextrakten aus Mäuseorganen.

\begin{tabular}{|c|c|c|}
\hline \multicolumn{3}{|c|}{ Streptococcus haemolyt. } \\
\hline & $\begin{array}{c}\text { Grad der Verdünnung } \\
\text { des Extraktes in } 5 \mathrm{ccm} \\
\text { Normosal mit I\% } \\
\text { Glyzerin v. . } \% \text {. Natr. } \\
\text { asparag. }\end{array}$ & Zahl der Kolonien \\
\hline $\begin{array}{c}\text { Die Mäuse wurden schwer } \\
\text { erkrankt getötet, nachdem } \\
\text { sie } 48 \text { Stunden vorher mit } \\
\text { Friedländerstamm T. ge- } \\
\text { impft waren. }\end{array}$ & $0,2 \%$ & $\left.\begin{array}{l}287,551 \\
386,952 \\
323,051\end{array}\right\} \dagger$ \\
\hline $\begin{array}{l}\text { Die Mäuse wurden gesund } \\
\text { getötet und sofort verar- } \\
\text { beitet }\end{array}$ & $0,2 \%$ & $\left.\begin{array}{l}3,200 \\
3,000 \\
3,000\end{array}\right\} \dagger$ \\
\hline & $\begin{array}{l}\text { Kontrolle ohne } \\
\text { Extrakt, bebrütet }\end{array}$ & $I, 800$ \\
\hline & $\begin{array}{c}\text { Kontrolle ohne } \\
\text { Extrakt, unbebrütet }\end{array}$ & 3,800 \\
\hline
\end{tabular}

*) Die Alkoholextrakte der mit Milchsäure versetzten Organe waren biuretpositiv. **) Als ganz gesund wurden Mäuse nur angesehen, wenn die Temperatur über $37^{\circ}$ war. Bei der Sektion wurden alle Organe genau geprüft.
†) Drei Zählplatten der gleichen Verdünnung. 
Daß man aus Friedländerreinkulturen allein schon aktivierendeStoffe gewinnen kann, geht aus folgenden Versuchen hervor:

Es wurden 30 große mit Agar ausgegossene Drigalskischalen mit einem gut wachsenden Friedländerstamm beimpft. Am nächsten Tage wurden die gut bewachsenen Platten durch absoluten Alkohol mit Hilfe des Glasspatels abgeschwemmt. Die Alkoholabschwemmung wurde am Rückflußkühler 20 Minuten gekocht, dann filtriert und im Faust-Heim eingetrocknet. Das Extrakt wurde mit destilliertem Wasser verrieben, filtriert, neutralisiert (Lackmus) und wiederum eingetrocknet. In gleicher Weise wurden unbewachsene, auf Sterilität geprüfte Platten des gleichen Agars ebenso behandelt. Sodann wurden die Extrakte, wie bereits oben beschrieben, in der in der Tabelle angegebenen Verdünnung kurz im strömenden Dampf sterilisiert und in gleicher Weise mit der gleichen Öse einer, I8stündigen gleichmäßig bewachsenen Streptokokken-Bouillonkultur beimpft, sodann nach I6-I7stündigem Bebrüten bei $37^{\circ}$ Blutagarzählplatten gegossen.

\section{Tabelle IV.}

\begin{tabular}{|c|c|c|c|}
\hline \multicolumn{4}{|c|}{ Streptococcus h aemolyt. } \\
\hline $\begin{array}{l}\text { Grad d. Verdiinnung } \\
\text { des Extraktes in } \\
5 \mathrm{ccm} \text { Normosal mit } \\
\mathrm{I} \% \text { Glycerin u. I } \% \\
\text { Natr. asparag. }\end{array}$ & $\begin{array}{l}\text { Zahl der Kolon en } \\
\text { bei Verwendung von } \\
\text { Extrakt von Fried- } \\
\text { Iänderkulturen }\end{array}$ & \begin{tabular}{|} 
Zahl der Kolon' en \\
bei Verwendung von \\
Extrakten von Ab- \\
schwemmungen \\
ster'ler Agarplatten
\end{tabular} & $\begin{array}{c}\text { Kontrollen obne } \\
\text { Extrakt }\end{array}$ \\
\hline $0,2 \%$ & $\left.\left.\begin{array}{l}228,384 \\
189,334 \\
213, \text { OOI }\end{array}\right\} *\right)$ & $\left.\begin{array}{l}5,400 \\
4,000 \\
2,600\end{array}\right)$ & bebrütet \\
\hline $0,02 \%$ & $\left.\left.\begin{array}{l}9,600 \\
9,400 \\
5,400\end{array}\right\} *\right)$ & $\left.\left.\frac{-}{200}\right\} *\right)$ & $\begin{array}{c}\text { unbebrütet } \\
400\end{array}$ \\
\hline
\end{tabular}

Der auffallende Unterschied sei durch die beigegebene Photographie festgehalten. Sie gibt auf dem beschriebenen Wege gewonnene Zählplatten der in o,2 pro mille Extraktverdünnung gewachsenen Streptokokken wieder. (Gegossen wurden die Zählplatten in der Verdünnung $\mathrm{I}:$ Ioo.) Abb. I.
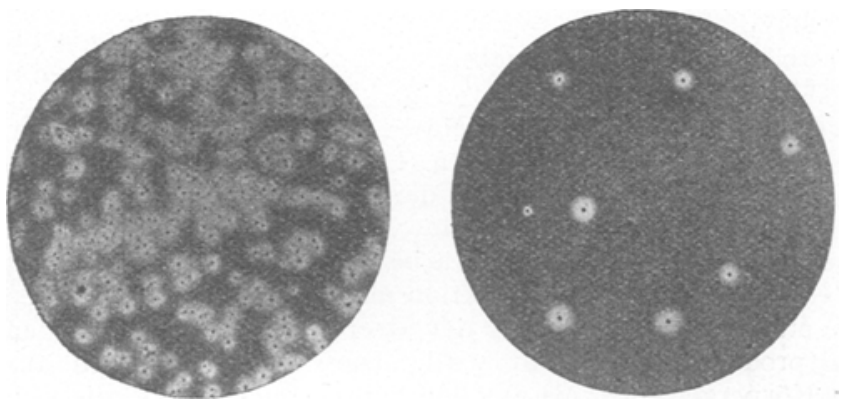

Abb. 1 .

Schließlich sei noch eine Konzentrationstabelle angeführt, aus der $\mathrm{zu}$ entnehmen ist, $\mathrm{da} \beta$ die aktivierenden abiureten Extrakte schon in recht großen Verdünnungen eine Vermehrung der Parasiten auf Nährmitteln, auf welchen sie sonst nicht zu wachsen vermögen, bedingen. Bei stärkeren Konzentrationen wird ein Optimum erreicht und bei noch höheren Konzentrationen ist eine deutliche Wachstumshemmung festzustellen.

Tabelle V.

Wachstumszahlen mit wässerigen Auszügen von Alkoholextrakten aus Meerschweinchenorganen nach i 7 stünd. Verweilen bei $37^{\circ}$,

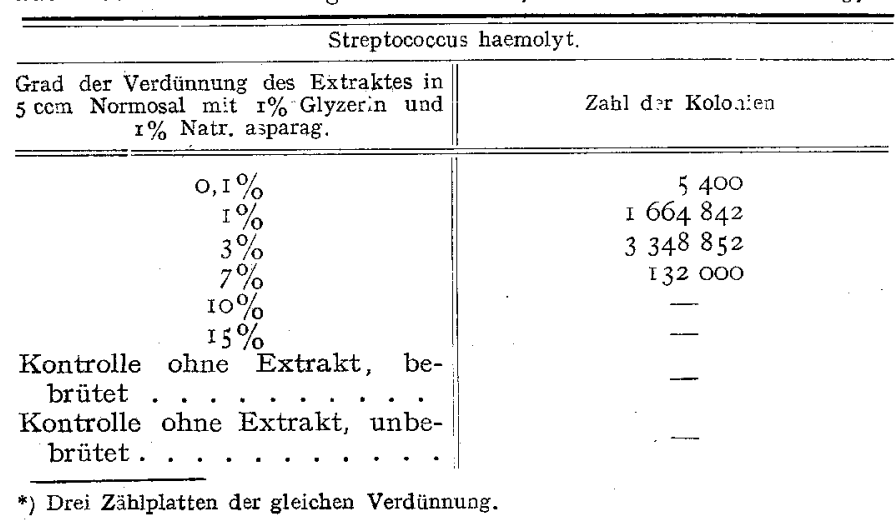

An anderer Stelle habe ich ausgeführt*), daß auch hier wie auf vielen Gebieten, eine Vielheit der Ursachen vorliegen mag. Strukturchemisch ist die Vermehrung der auf den Körper eingestellten Parasiten infolge Anwesenheit geeigneter, dem Körper entnommener Gruppen verständlich, physikalisch dürfte in bestimmten Konzentrationen eine Dispersitätsvermehrung und infolgedessen eine Erhöhung des Stoffwechsels in Frage kommen. In höheren Konzentrationen sind an geeigneten Objekten Fällungserscheinungen $\mathrm{zu}$ beobachten, so daB Dispersitätsverminderungen verständlich sind.

Aus folgenden Versuchen geht hervor, da $B$ durch unsere abiureten Extrakte nicht nur die Vermehrung als solche, sondern auch andere meBbare Fermentfunktionen bestimmter Parasiten beeinflußt werden (Kohlenhydratstoffwechsel, Giftbildung usw.). Bemerkenswert ist, da $\beta$ diese Beeinflussung von der Konzentration des wirksamen Extraktes abhängt. Das Optimum der Wirkung ist sowohl für die verschiedenen Stämme als auch für die verschiedenen Fermentfunktionen verschieden:

Streptokokkenstämme wachsen in Tyrodelösung nicht, im Gegenteil, die eingesäten Keime gehen darin in 48 Stunden zugrunde, wie wir an der Hand von Zählplatten bei vielen Stämmen feststellen konnten. Setzt man $2 \%$ unserer abiureten Extrakte zu Tyrodelösung ohne Traubenzucker, so bekommt man erhebliche Streptokokkenzahlen. Bei Zusatz von I\% Traubenzucker dagegen sind die Streptokokkenzahlen nach $4^{8}$ Stunden Wachstums gering, trotz Vorhandensein von Säure. Wir sehen also, daß Keimzahl und Säuregrad durchaus nicht konform gehen. Eine elektive Beeinflussung des Kohlenhydratabbaues liegt zweifellos vor.

Von besonderem Interesse ist ferner die Beeinflussung der Giftbildung durch unsere Extrakte. Auch hier ist eine Steigerung nur bei bestimmten Konzentrationen, die wieder nach den verschiedenen Stämmen schwanken, festzustellen. Bei höheren Konzentrationen findet Herabsetzung statt.

Folgende Abbildung gibt eine nach der intracutanen Methode Römers mit Diphtherietoxin behandelte Meerschweinchenhaut wieder. Links wurde die Injektion mit Diphtheriebouillonkultur vorgenommen, rechts waren die Bacillen in 2 proz. Extrakt-Tyrodelösung gewachsen. Thre Vermehrung war in dieser Konzentration des Extraktes zwar auch herabgesetzt und ihre Formen waren verändert, doch war die Giftbildung in viel höherem Maße geschädigt als die Vermehrung. Auf der rechten Seite war die Injektionsmenge um das Vierfache gegenüber links gesteigert, so daß dadurch

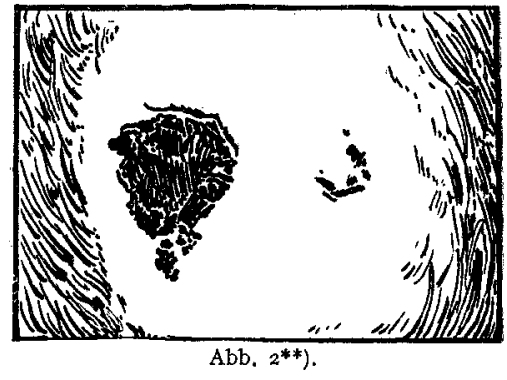
eine absolut größere Bacillenmenge als an der linken Stelle injiziert worden war. Trotzdem ist die Toxinwirkung an dieser Stelle ganz gering.

Wir gewinnen durch derartige Versuche, die von mir schon seit langem angestellt wurden und zum Teil auch schon veröffentlicht worden sind, neue Einblicke in das Wesen vieler Virulenz- und Immunitätserscheinungen.

Wie ich bereits im Jahre I920 in Nr. $3^{8}$ der Münch. med. Wochenschr. S. Io86 mitgeteilt habe, gelang es mir, ,thermostabile, die Infektionserreger aktivierende abiurete Wuchsstoffe aus dem Tierkörper auszuziehen" und zu zeigen, daß die gleichen Substanzen, welche eine Aktivierung der Infektionserreger bedingen, je nach der Verteilung, auch ihre Vermehrung und den übrigen Stoffwechsel herabsetzen können***).

*) Dtsch, med. Wochenschr. I92r, S. 885 und Referat auf dem Kongreß der deutschen Dermatolog. Ges. Hamburg, Mai I921, Kongreßbericht Arch. f. Dermatolog. u. Syphil. 83, 160. 1922,

*) Die Abbildung 2 ist dex Inaugural-Dissertation von IRMGARD v. SODEN „Eiweißfreie alkoholisch-wäBrige Extrakte aus dem tierischen Körper und ihr Einflu $B$ auf Biologie und Morphologie von Mikroorganismen“, Erlangen 1922, entnommen.

***) S, auch Nr. 3I der Dtsch. med. Wochenschr. I92I S. 885 und Referat vor der Dtsch. Dermatolog. Gesellsch, in Hamburg, Mai I92I, Arch. f. Dermatolog. u. Syph. 183, 160. I921. 
Diese Herabsetzung hängt von zwei Faktoren ab: von der Konzentration der Substanzen und von der Empfindlichkeit der Fermentfunktion. Man sollte, wie ich in Nr. 4 der Münch. med. Wochenschr. I922 ausgeführt habe, diesen abiureten Substanzen, die nach den verschiedensten Richtungen jetzt mit chemischen und physikalischen Mitteln durchforscht werden können, nicht in grob schematischer Weise lediglich eine reizende Wirkung zuschreiben. Über die Berechtigung des Ausdruckes ,,Aktivierung " auf diesem Gebiete siehe meine Diskussion mit A. BIER in de: Münch. med. Wochenschr. I92I, S. 365 .

. Neuerdings weisen A. v. WASSERMANN und M. FICKER ${ }^{10}$ ) in einer kurzen Mitteilung auf noch zu veröffentlichende Versuche von KoJima hin. Nach diesem wird durch Zufügen von Aktivatoren $\mathrm{zu} 2 \%$ Traubenzuckerbouillon die vermehrte Produktion einer sehr akut wirkenden giftigen Substanz seitens des Fränkelbacillus bewirkt.

Die Autoren knüpfen hieran die Anschauung, daß das Vorhandensein derartiger Aktivierungen im Darmkanal zu schweren Störungen des Allgemeinbefindens führen kann und daß in solchen Fällen die ,mechanische Entfernung der Darmingesta, welche als Aktivator die eigentliche Ursache darstellen, weit bessere Ergebnisse erzielen muß, als der Versuch durch eine immer problematische Darmdesinfeletion auf die Darmbakterien direkt wirken zu wollen".

Durch diese Ausführungen werden unsere über die Aktivierung von Streptokokken gewonnenen Anschauungen und Befunde bestätigt und erweitert (s. a. A. SchittenHELM²).

Chemische Charakterisierungen der im Darmkanal gefundenen aktivierenden Spaltprodukte wurden bisher nicht mitgeteilt. Es wäre $z$. B. interessant $z u$ wissen, ob schon abiurete Substanzgemische auch hier für die Aktivierung genügen. Auch sind Konzentrationsversuche noch nicht bekannt geworden.

Aus unseren Befunden mit Streptokokken möchte ich jedoch den Schluß ziehen, daß auch in bezug auf die Darmflora das gleiche gilt wie in bezug auf die Beeinflussung des Fermentstoffwechsels der Streptokokken durch abiurete Körperextrakte. In höheren Konzentrationen werden auch hier die einzelnen Fermentfunktionen gelähmt werden.

Ich möchte deshalb die praktischen. Schlußfolgerungen der beiden Autoren auf Grund unserer älteren Erfahrungen auf diesem Gebiete anders formulieren:

Die gleichen Substanzen, welche die Infektionserreger aktivieren, können in höherer Konzentration auch als Schutzstoffe wirken. Oft ist also ihr Vorhandensein im Körper wichtig und ihre Entfernung nicht erwünscht. Gelingt sie nicht vollständig, was in der Praxis nicht selten der Fall sein dürfte, so wirken die geringen zurückbleibenden Reste auf gewisse Bakterien ,,aktivierend".

Auch hierfür lassen sich praktische Beispiele anführen.

Literatur: 1) Petersen, W. F., Protejn Therapy etc. The Macmillan Company. New York i922. - ${ }^{2}$ ) A. Schitrenhelm Theorie und Praxis der Proteinkörperwirkung. Referat auf der 9. Versammlung der freien Vereinigung für Mikrobiologie Pfingsten 1922. Zentrbl.f. Bakt. etc. u. Med. Klin. 1922, S. 949 u. Münch. med. Wochenschr. 1921, S. 35 I. $-{ }^{3}$ ) W.WEICHaRDT, Ergebnisse der Hygiene. (Berlin, Julius Springer). 5, 275. I922. - ${ }^{4}$ ) R. ScHмIDT, Ergebnisse d. ges. Med. 3, S. $56 .-5)$ H. Freund, Med. Klin. I920, S. 437; Arch. f. exp. Pathol. 1. Pharmakol. 93, H. I-3, S. 92. 6) Starkenstein, Münch. med. Wochenschr. 19I9, S. 205. 7) EDELMANN, Wien. klin. Wochenschr. I9r7, S. 497. - 8) KYRLE, Wien. klin. Wochenschr. I9I 7, S. 707. - ${ }^{9}$ ) W. WEICHARDT, Münch. med. Wochenschr. 1922, S. I07; I92 I, S. 365 ; I92I, S. 39; I920, S. 1085 ; 1920, S. 91 ; I919, S. 289 ; 1918, S. 581 ; I9I 5, S. I 525 ; Zeitschr. f. d. ges. Neurol. u. Psych. I9I4, 22, S. 586; Ergebnisse d. Hyg. 5, S. 275 ; Dtsch. med. Wochenschr. 1921, S. 885 ; Berl. klin. Wochenschr. I921, S. $872 .-10$ ) A. v. WASSERMANN Ficker M., diese Wochenschr. I922, S. I 159.

\section{DIE ENTEROTROPISCHE HARNSÄURE. [ $Z_{\text {weite }}$ Mitteilung $)^{1}$.] \\ Von}

Prof. Theodor Brugsch und Dr. Julius Rother.

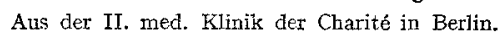

Wer sich von der Harnsäure in der Galle überzeugen will, möge folgendes Verfahren anwenden:

$50 \mathrm{~cm}$ Gallenblaseninhalt von Leichen werden mit $200 \mathrm{ccm}$ Wasser und $5 \mathrm{ccm}$ konz. Schwefelsäure versetzt; etwa vorbandene grobe Schleimmassen werden vorher abzentrifugiert oder durch Stehenlassen sedimentiert. Man kocht fünf Stunden am Rückflußkühler, macht noch warm mit Natronlauge schwach alkalisch und dann mit Essigsäure schwach sauer. Der (manchmal dunkelgrüne, manchmal schwarzbraune) Niederschlag wird abfiltriert und hei $\beta$ ausgewaschen. Das Filtrat wird mit etwa io g Natriumacetat und etwa $3 \mathrm{~g}$ Natriumbisulfit versetzt und zum Sieden erhitzt. Beim Zusatz von etwa $20 \mathrm{ccm}$ Io proz. Kupfersulfatlösung fällt nunmehr ein Niederschlag aus, der nach dreiminutenlangem Sieden abfiltriert, mit heißem Wasser gut ausgewaschen, vom Filter abgespritzt und dann nach Zusatz von etwa $\mathrm{I} \mathrm{ccm} \mathrm{konz.} \mathrm{Salzsäure}$ durch Einleiten von Schwefelwasserstoff unter Erwärmen zerlegt wird. Nach vollständigem Verjagen des überschüssigen Schwefelwasserstoffes (Kontrolle durch Bleipapier) wird das Filtrat auf einige Kubikzentimeter eingedampft. Die Harnsäure krystallisiert genau wie aus dem Harn schön aus und ist leicht als solche zu identifizieren.

Der gleiche Versuch gelingt, wenn man durch Hepaticusdrainage erhaltene Lebergalle vom Menschen demselben Verfahren unterwirft. Wir haben die Harnsäure aus $300 \mathrm{ccm}$ Fistelgalle dargestellt.

Naturgemäß ist dieses Verfahren nur ein präparatives und kein quantitatives, da durch Aufschließen mit Schwefelsäure nach fünfstündigem Kochen Harnsäure zerstört wird. So fanden wir beispielsweise durch fünfstündiges Erhitzen von $20 \mathrm{mg}$ Harnsäure in $200 \mathrm{ccm}$ Wasser und $5 \mathrm{ccm}$ Schwefelsäure nach diesem Verfahren nur etwa den dritten Teil, von Io mg Harnsäure nur etwa den fünften Teil wieder. (Colorimetrische Analysen.)

Trotzdem gelang es uns so, präparativ aus der Fistelgalle rund $50 \mathrm{mg}$ in Iooo Galle aufzufinden; aus der Blasengalle rund $200 \mathrm{mg}$. Das sind nur Minimalwerte; der wirkliche Wert muß weit höher liegen.

Die Frage der Harnsäureausscheidung mit der Galle ist eine derart grundlegende, daß unser Bestreben dahin gerichtet sein mußte, hier wenigstens annähernde Vorstellungen über die Größe der enterotropischen Harnsäure zu gewinnen. Vier Wege mußten beschritten werden: Die Untersuchung der Blasengalle, der Fistelgalle, der Duodenalgalle und der Faeces. Man kann die Blasengalle nicht einfach mit der Fistelgalle und mit der Duodenalgalle vergleichen, denn die Blasengalle ist eingedickte Galle, die Duodenalgalle kann eingedickte Galle sein oder auch verdünnte Galle durch Beimischung von Magendarmsaft. Um hier einen Ausweg zu finden, haben wir die Fiktion der reduzierten enterotropischen Harnsäure eingeführt. Wir fanden mittels der HIJMANS vaN DEN BERGH schen Colorimetrie den Farbstoffgehalt der Blasengalle durchschnittlich I : I000, der Fistelgalle I : to ooo, der Duodenalgalle zwischen I : 10000 und I : 200 ooo. Man darf wohl nach unseren Erfahrungen aus der Duodenalsondierung auf 24 Stunden berechnet einen Duodenalgallenwert von I0oO $\mathrm{ccm}$ mit einem Farbstoffgehalt von $\mathbf{o}, \mathbf{I} \mathbf{g}$ als die Norm ansehen. Auf diesen Wert ist der Harnsäurewert der Fistelgalle oder Blasengalle oder Duodenalgalle zu reduzieren, solange wir nicht die Möglichkeit haben, an Gallenfistelträgern mit vollständigem Abschluß nach dem Darm hin die Gallensekretion absolut zu fassen.

Das schwierigste war nun die Methodik der Harnsäurebestimmung in der Galle. Nach vielen Vorversuchen ist es uns gelungen, eine Methode zu finden, mit Hilfe deren es möglich ist, nach Fällung der Harnsäure mit Silber die Bestimmung colorimetrisch durchzuführen. Für die konzentriertere Blasengalle haben wir allerdings Versager dieser Methode zu verzeichnen, was vermerkt werden muB.

Die Methode besteht im Zusatz von einigen ccm konz. Ammoniaks zu etwa $50 \mathrm{ccm}$ Duodenal- oder Fistelgalle, deren Farbstoff-

3) Vgl. die erste Mitteilung: Diese Żeitschrift Nr. 30, S. 1495 (I922). ; 